\title{
A novel decrystallizing protein CXEXL22 from Arthrobotrys sp. CX1 capable of synergistically hydrolyzing cellulose with cellulases
}

\author{
Rong Li, Yunze Sun, Yihao Zhou, Jiawei Gai, Linlu You, Fan Yang, Wenzhu Tang and Xianzhen Li
}

\begin{abstract}
A novel expansin-like protein (CXEXL22) has been identified and characterized from newly isolated Arthrobotrys sp. CX1 that can cause cellulose decrystallization. Unlike previously reported expansin-like proteins from microbes, CXEXL22 has a parallel $\beta$-sheet domain at the $N$ terminal, containing many hydrophobic residues to form the hydrophobic surface as part of the groove. The direct phylogenetic relationship implied the genetic transfers occurred from nematode to nematicidal fungal Arthrobotrys sp. CX1. CXEXL22 showed strong activity for the hydrolysis of hydrogen bonds between cellulose molecules, especially when highly crystalline cellulose was used as substrate. The hydrolysis efficiency of Avicel was increased 7.9-fold after pretreating with CXEXL22. The rupture characterization of crystalline region indicated that CXEXL22 strongly binds cellulose and breaks up hydrogen bonds in the crystalline regions of cellulose to split cellulose chains, causing significant depolymerization to expose much more microfibrils and enhances cellulose accessibility.
\end{abstract}

Keywords: Cellulose depolymerization, Crystalline region, Expansin-like protein

\section{Introduction}

Cellulose is a polymer consisting of glucose line linked by $\beta-1,4$ bonds and contains crystalline region and amorphous region. Many studies in the past 50 years focused on the dissolution and degradation of cellulose as the renewable biomass and biomaterial (Guo et al. 2018; Ragauskas et al. 2006). However, the conversion of cellulose into fuel is still limited because of its physical properties, especially the degree of polymerization, crystallinity and accessibility (Arantes and Saddler 2010; Wilson 2009). The cellulose polymerization is formed by the agglomeration of microfibrils (dimeter around $10 \mathrm{~nm}$, consisting of cellulose chains). The intermolecular and intramolecular hydrogen bonds tightly packed cellulose

*Correspondence: xianzhen@mail.com

School of Biological Engineering, Dalian Polytechnic University,

Gangjingqu, Dalian 116034, China chains result in various ordered crystalline arrangements, forming cellulose crystalline region with high crystallinity. The cellulose accessibility is the most important factor for the rate and extent of cellulose hydrolysis, reflecting the available area for aqueous solution and cellulase to accessing and hydrolyzing cellulose chains, which is strongly influenced by polymerization and crystallinity (Arantes and Saddler 2010; Lindman et al. 2010). Thus, cellulose depolymerization and decrystallization must be improved to promote cellulose accessibility (Arantes and Saddler 2010; Lindman et al. 2010).

Ionic liquid and traditional organic solvents have been used in cellulose decrystallization and depolymerization. However, their application is limited by their environmental hazard, recovery problem and high cost (Rinaldi et al. 2008; Tadesse and Luque 2011; Chen et al. 2015). Enzymatic degradation is an environmental-friendly process of accessing and binding the cellulose chain 
to hydrolyze and diffuse cellulose (Sharma et al. 2019; Ravindran and Jaiswal 2015). Some accessory proteins, such as lytic polysaccharide monooxygenase (LPMO), expansin, expansin-like protein, swollenin and loosenin, can disrupt and lytic insoluble microfibril polymerization as cellulose pretreatment (Wilson 2012; Liu et al. 2015). These accessory proteins exhibit synergism with traditional cellulase to improve the cellulose degradation but cannot hydrolyze cellulose directly (Wilson 2012; Liu et al. 2015; Sabbadin et al. 2018). LPMOs produce several gaps on the surface of cellulose by oxidation, which promotes the accessibility of the cellulase to boost cellulose degradation (Villares et al. 2017).

Expansin is a wall-loosening protein obtained from plant. Various expansin-like genes with similar sequence homology and functions as expansin have been found in different microbes and other organisms that live in soil or produce cellulose (Liu et al. 2015; Cosgrove 2000; Georgelis et al. 2015). Recent reviews have detailed their discovery, classification, phylogenetic distribution and evolution. In addition, expansin-like from microbes show some similarity to expansin from plant: (i) these proteins loosen hydrogen bonds between polysaccharide chains without detectable hydrolytic activity; (ii) these proteins contain a double $-\Psi \beta$-barrel fold (DPBB) domain D1 at $\mathrm{N}$-terminal and a $\beta$ sandwich fold domain D2 at C-terminal; and (iii) these proteins have an acidic optimum to extension activity. These points are further supported by the fact that expansin-like proteins from microbes have evolved from a plant ancestor by horizontal gene transfer (HGT) (Nikolaidis et al. 2013). However, the expansinlike protein derived from nematodes is likely an example of independent evolution. Nematodes secrete a complex of hydrolytic enzymes to overcome the barrier presented by their host cell wall, which aid in root penetration and migration. The expansin-like protein from nematodes has a GH45 domain at its C-terminus, a structure indicative of separate evolutionary origin, which is inconsistent with canonical expansins (Liu et al. 2015; Sabbadin et al. 2018; Georgelis et al. 2015; Cosgrove 2015, 2017). Moreover the domain D1 of expansin and expansin-like protein resembles the catalytic domain of the GH45 without catalytic residues that is normally required for GH45 hydrolytic activity. The domain D2 forms a nearly flat surface populated with aromatic residues, resembling some carbohydrate-binding modules (CBM) to bind cellulose (Liu et al. 2015; Georgelis et al. 2015).

Arthrobotrys sp. CX1 is a nematicidal fungal strain that gelatinizes the filter paper cellulose. It was isolated and identified from soil sample with the rotted wood (Lan et al. 2016). It can gelatinize the filter paper cellulose causing decrystallization with high water imbibition, but cannot degrade cellulose. Thus, the cellulose decrystallization and depolymerization by Arthrobotrys sp. CX1 can facilitate water penetration in the cellulose but not cellulose degradation. In this work, we obtained a novel expansion-like protein (CxEXL22) from Arthrobotrys sp. CX1 that can cause cellulose decrystallization for the first time. Differing from proteins previously reported from microbes, CxEXL22 is composed of a parallel $\beta$-sheet domain at the $\mathrm{N}$ terminal and a DPBB expansin domain at the $\mathrm{C}$ terminal. The phylogenetic relationship showed the CxEXL22 was directly grouped with expansin-like proteins from nematode. It was indicated the expansin-like protein genetic transfers occurred from nematode to nematicidal fungal Arthrobotrys sp. CX1. The parallel $\beta$-sheet domain of the CxEXL22 forms the hydrophobic surface to bind the cellulose, although the domain is different from the $\beta$-sandwich fold domain D2 of the previous reports indicated expansin-like proteins. CxEXL22 was expressed and biochemically characterized. The rupture of the crystalline regions was examined with X-ray diffraction (XRD), scanning electron microscopy (SEM) and Fourier transform infrared spectrometer (FTIR) after the pretreatment with CxEXL22. Results suggested that CxEXL22 had a potential application in constructing unique nanocellulosic structures because of its capacity to induce cellulose depolymerization.

\section{Materials and methods}

\section{General information}

All chemical reagents in this study were obtained from commercial supplier Sinopharm Chemical Reagent Co. Ltd., Shanghai, China. Cellulosic substrates and commercial cellulase were purchased from Sigma-Aldrich, Mo, USA. Phosphoric acid-swelling cellulose (PASC)) was prepared from Avicel PH -101 (Kuo and Lee 2009). Fungal strain of Arthrobotrys sp. CX1 was isolated from the campus of Dalian Polytechnic University, China, that was cultured as previously described (Lan et al. 2016). Pichia pastoris X-33 and vector $\mathrm{pPIC} \alpha \mathrm{A}$ were purchased from Invitrogen.

\section{Analysis and cloning of CxEXL22}

The sequences of 15 full-length expansins and expansinlike proteins from different microbes, plants and nematode were aligned and a neighbor-joining phylogenetic tree was constructed using Molecular Evolutionary Genetics Analysis (MEGA) 6.0 software (Tamura et al. 2013). A conserved domain was identified using the Conserved domain Database. Total RNA from Arthrobotrys sp. CX1 was extracted using Trizol method that was grown in the presence of the filter paper (Lan et al. 2016). The CxEXL22 cDNA sequence was amplified from a sample of total RNA of Arthrobotrys sp. CX1 by reverse transcription coupled-PCR with the following primers: 
CxEXL22-T forward primer 5'-GACATGTTACAA AAGCGGG-3', CxEXL22-T reverse primer 5'-TTAGAC GTAATCCCAGGTG-3'. The 633-bp PCR fragment was purified and cloned into the pMD 18-T Vector (Takara) resulting in PMD 18-CXEXL22, and its sequence was confirmed. The pMD 18-CXEXL22 as template was amplified by restriction-free cloning using the following primers: 5'-CTAAAGAAGAAGGGGTATCTCTCGAGAAAA GACATGTTACAAAAGCGGG-3'and5'-GAGTTTTTG TTCTAGAAAGCTGGCGGCCGCTTAGACGTAATC CCAGGTG- $3^{\prime}$ and constructed with pPICZ $\alpha A$ (Van Den Ent and Löwe 2006). Recombinant plasmid pPICZ $\alpha A-$ CXEXL22 was transformed into the host Escherichia. coli DH10B and its sequence was confirmed by restriction analysis and DNA sequencing. The nucleotide sequence of CxEXL22 has been deposited in the Genbank database under submission MN138044, which is without the signal peptide.

\section{Modeling the structure of CxEXL22}

The CxEXL22 was used for homology modeling using the Phyre2 web server (Kelley et al. 2015). The structure of CxEXL22 is modeled based on EXLX1 [PDB ID: 3D30] and Clavibacter michiganensis expansin [PDB ID: 4JCW]. The sequence identity is $26 \%$ between CxEXL22 with EXLX1, and it is 36\% between CxEXL22 with Clavibacter michiganensis expansin.

\section{Expression and purification of CxEXL22}

$P$. pastoris $\mathrm{X}-33$ was electrically transformed with linearized vector pPICZ $\alpha \mathrm{A}-C x E X L 22$, and transformants were selected by Zeocin $(100 \mu \mathrm{g} / \mathrm{mL})$. The CXEXL22 gene in the transformants was confirmed by PCR using yeast genomic DNA as template to be transformed into $P$. pastoris X-33. The CxEXL22 protein was expressed in $P$. pastoris $\mathrm{X}-33$ in accordance with the manufacturer's protocols (Invitrogen).

The crude supernatant was collected by centrifugation at $8000 \mathrm{rpm}$ for $5 \mathrm{~min}$ after cultured. The recombinant CXEXL22 was purified by concentrating the supernatant 15 -fold by ultrafiltration at $4000 \mathrm{rpm}$ for $40 \mathrm{~min}$ using centrifugal filter devices (exclusion size $10 \mathrm{kDa}$, Millipore), and then applying it to a HisTrap HP column (5 mL, GE Healthcare) pre-equilibrated with phosphate buffer (20 mM pH 6.0). The fractions were collected and checked by SDS-PAGE.

\section{CXEXL22 and commercial cellulase synergistically acted on substrates}

CxEXL22 was used to measure activity with final concentrations of $100 \mathrm{mM}$ buffer, $5 \mathrm{mg}$ substrate, $5 \mu \mathrm{g}$ CxEXL22 and $0.3 \mathrm{U}$ commercial cellulase in a $5 \mathrm{~mL}$ reaction at $\mathrm{pH}$ 6 for $40 \mathrm{~h}$ at $30^{\circ} \mathrm{C}$ with $160 \mathrm{rpm}$. According to the literature, the commercial cellulase was loaded into the reaction mixture (Lee et al. 2010). The concentration of the released reducing sugar was determined using the DNS method described previously (Zhang et al. 2009). All assays were performed in triplicate.

The reaction was treated at different temperatures $\left(30,40,50,60\right.$, or $\left.70{ }^{\circ} \mathrm{C}\right)$ to determine the effect of temperature on CxEXL22. The reaction was terminated and reducing sugars were determined as explained above. For the whole range of buffer, the reducing sugar was determined in the reaction at the $\mathrm{pH}$ range of 3.0-7.0. The reaction was treated at different cellulase loading (0.05$0.45 \mathrm{U} / \mathrm{ml}$ ) to determine cellulase loading to affect the synergetic action of CxEXL22.

\section{Analysis with XRD, IFTR and SEM}

CxEXL22 was used to measure activity with final concentrations of $100 \mathrm{mM}$ buffer, $5 \mathrm{mg}$ substrate(cotton, filter paper and Avicel), $5 \mu \mathrm{g}$ CxEXL22 in a $5-\mathrm{mL}$ reaction at $\mathrm{pH} 6$ for $48 \mathrm{~h}$ at $30^{\circ} \mathrm{C}$ with $160 \mathrm{rpm}$. Control experiments without CxEXL22 or using $\mathrm{H}_{2} \mathrm{O}$ were also performed under the same conditions as mentioned above. For microscopic observations, the samples were washed three times by ultrasonic and then dried to completely remove all residual solvent. Then the substrates were visualized by SEM (JSM-7800F and JSM-6460LV, JEOL, Japan). The changes in chemical bonds and functional groups were detected by FTIR (Spectrum two, PerkinElmer, China) and changes in cellulose crystallinity and structure were determined by XRD (XRD-7000S, Shimadzu, Japan) as described previously (Lan et al. 2016).

\section{Results and discussion}

Sequence analysis of an expansin-like protein from Arthrobotrys sp. CX1

Arthrobotrys sp. CX1 was isolated from a soil sample with rotted wood collected from the campus of Dalian Polytechnic University, China, which can disrupt cellulose chains to make the filter paper translucent (Lan et al. 2016). Our interest is to reveal fungal-derived enzymes

(See figure on next page.)

Fig. 1 Sequence and phylogenetic analysis of CXEXL22. A The predicted domain organization of CXEXL22; $\mathbf{B}$ sequence alignment of CXEXL22, BsEXLX1 from Bacillus subtilis (GenBank: AHH91628.1), EXPB1 from Zea mays (NCBI: XP_008659972.2), and ScExIx1 from Schizophyllum commune (GenBank: AKN44794.1); C phylogenetic tree of CXEXL22 
(A)

27

89

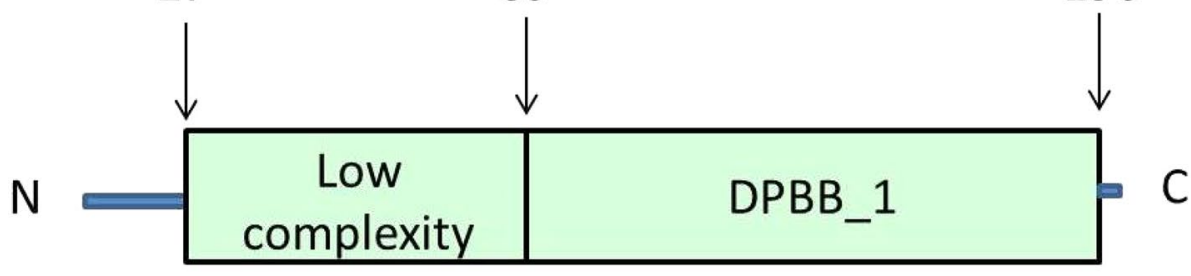

CXEXL22

BSEX1X1

$\operatorname{SCE} \times 1 \times 1$

EXPB1

MLQKRDAVVYVNQEVLEIVVYTSTIWVAPPPPPNETPVPEPVVPTPTPEPVVAANETGVP

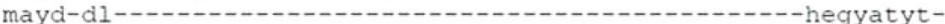
VHHT--

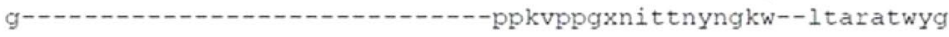
. . *

CXEXL22

BSEX1X1

$\operatorname{SCEX} 1 \times 1$

EXPB 1

VPVVI PPEPSPSPAPPVEEPPSSGGGGGTYTGKA-TFYDAGLGSCGETHSNSDMICALSK

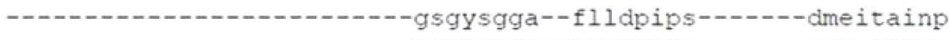
-

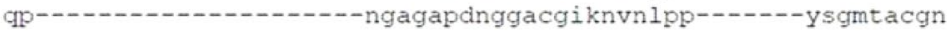
*. . * * : $\quad$ * .

CXEXL22 BSEX1X1

SCEX1X1 EXPB 1

CXEXL22

BSEx1X1

SCEXIX1

EXPB1

VTMALTAGPNPNLNPKCGTKIRV------MSASNPTGVIVTIVATCPGCLGPNDIDLTPA adlnygg---vkaalagsylev-----egpkgkttvyvt-- olypeg-argal o $1 \mathrm{spn}$ SEW-EGS------ASCGACLSV-----TGASGTATVMVV-- DKCPEC-EAGHIDLEQD

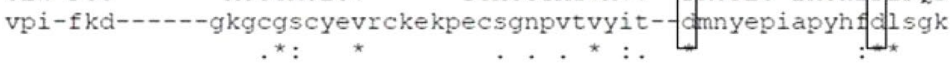

C

CXEXL22

BSEX1X1

SCEX1X1

tipklpesgtskaytvpghvgfpe

VA------VSSGSVVEGGSQE--

(B)

EXPB1

(C)

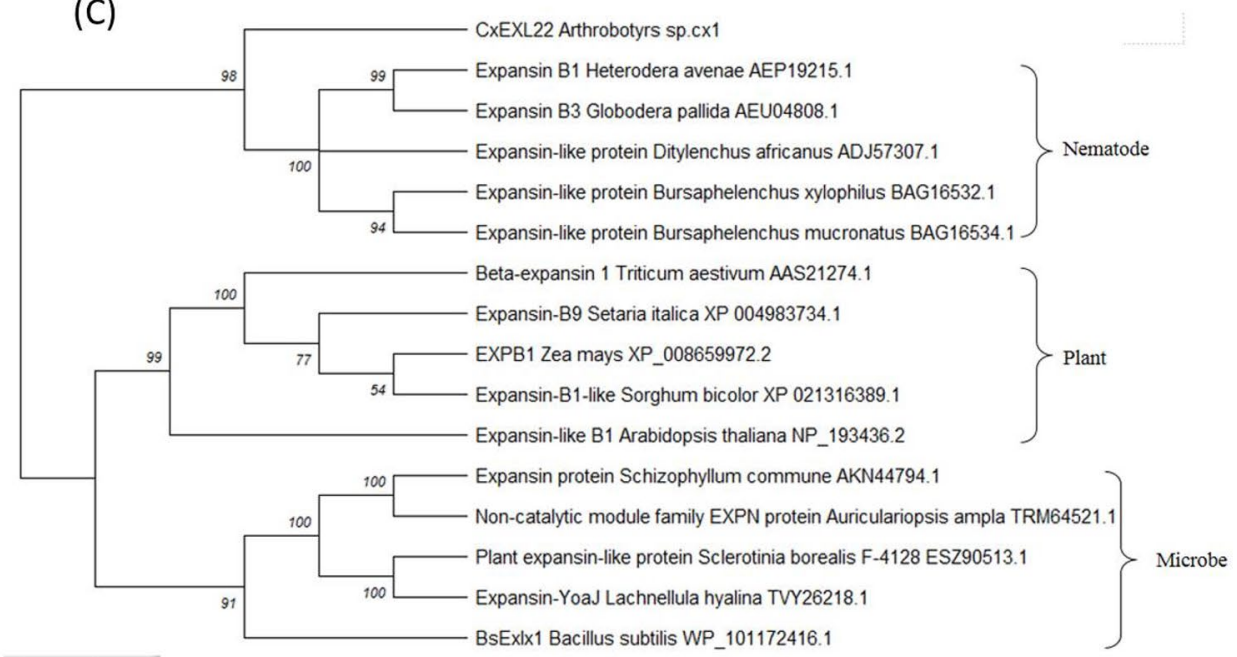

Fig. 1 (See legend on previous page.) 
responsible for this novel cellulolytic and cellulose destruction activity. On the basis of sequences analysis from cDNA library of Arthrobotrys sp. CX1, one sequence was termed "CxEXL22", which encoded a protein of 193 amino acid residues, containing the low complexity region (residues 27-89) and the DPBB region (residues 89-190), as predicted using the SMART server (Letunic et al. 2021) (Fig. 1A). The DPBB Domain of CXEXL22 was found to have sequence similarity with expansin-like proteins from fungal species Schizophyllum commune H4-8 [NCBI:XP_003029770.1] 47.12\%, Heterobasidion irregulare TC32-1 [NCBI:XP_009552773.1] 46.15\% and Gigaspora rosea [GenBank: RIB28841.1], annotated as an expansin-like family protein. It has been identified that these crucial residues D71 and D82 in BsEXLX1 for the expansin activity (Kerff et al. 2008). These key residues are all completely conserved in CxEL22, suggesting the domain DPBB in CxEL22 has the function same as the domain DBPP of expansin-like proteins (Fig. 1B). CxEXL22 contains DPBB expansin domain at C terminal, which is different from proteins previously reported from microbes. Most of the expansin-like proteins from microbes reported the special DPBB domain at the $\mathrm{N}$ terminal. However, those from nematode contain the DPBB expansin domain at the $C$ terminal (Georgelis et al. 2015; Nikolaidis et al. 2013; Cosgrove 2015).

The sequences of the expansin-related proteins were extracted and generated a phylogenetic tree, containing proteins from plants, microbe and nematode. An analysis of the phylogenetic relationship among these proteins showed that the sequences were separated into three clades (Fig. 1C). According to previous research of evolution of expansin-like proteins, these non-plant expansinlike proteins from bacteria and fungi originated in plant and transferred from plant to microbe by horizontal gene transfer, which revealed the evolution of plant and microbe species (Nikolaidis et al. 2013). Interestingly, the phylogenetic tree showed that the CxEL22 was directly grouped with expansin-like proteins from nematode. CxEL22 is identified from Arthrobotrys sp. CX1, which is a nematicidal fungal strain. The result strongly suggested that the expansin-like protein genetic transfers occurred from nematode to nematicidal fungal Arthrobotrys sp. CX1 (Additional file 1).

\section{Homology modeling of CxEXL22}

Most of expansins and expansin-like proteins consist of two domains (D1 and D2). The domain D1 is named DPBB domain to catalysis and the domain D2 is a $\beta$-sandwich fold to bind, resembling CBM (Liu et al. 2015; Georgelis et al. 2015) The CxEXL22 was used for fold recognition using the Phyer web server. Unlike the previously reported structure of expansin-like protein, the CxEXL22 protein was shown to have the parallel $\beta$-sheet domain at the $\mathrm{N}$ terminal and a DPBB domain at the $\mathrm{C}$ terminal (Fig. 2A). Previous reports indicated the expansin-like proteins to bind the cellulose via the hydrophobic surface of the domain D2, consisting of hydrophobic interactions of three linearly arranged aromatic residues (Liu et al. 2015; Cosgrove 2000; Georgelis et al. 2015). The parallel $\beta$-sheet domain of the CxEXL22 protein is different from that of the domain D2 of expansinlike protein. By comparing the $\beta$-sandwich-fold domain and the parallel $\beta$-sheet domain, both contain hydrophobic residues to form the hydrophobic surface, the $\beta$-sandwich-fold domain is flat surface and less hydrophobic area, while the parallel $\beta$-folded domain appears as a groove with more hydrophobic surface (Fig. 2B, C). The model suggests that the CxEXL22 binds to the cellulose through the groove on the hydrophobic surface of the parallel $\beta$-sheet domain, which is more effective than the $\beta$-sandwich-fold domain to contact the hydrophobic surface of cellulose microfibrils.

\section{Heterologous expression of CxEXL22 in P. pastoris}

The recombinant CxEXL22 was heterologously expressed in Saccharomyces cerevisiae and purified in one step with His-tag from culture supernatants to the more than $90 \%$ purity. Its molecular weight was calculated as approximately $50 \mathrm{kDa}$ by SDS-PAGE (Fig. 3A). DNA sequence analysis of the open reading frame revealed that it encoded a protein of 193 amino acid residues and a calculated mass of $21.7 \mathrm{kDa}$. This suggested the occurrence of the post-translational modification, especially glycosylation. The CxEXL22 were predicted N-glycosylation of Asn on residue 55 and 147 by the NetNGlyc 1.0 Server. In previous reports heterologously expressed expansin-like proteins also exhibit glycosylation in fungal hosts (Wang et al. 2014; Liu et al. 2014).

\section{Synergistic effect of CxEXL22 in cellulose hydrolysis}

Expansin-like proteins hydrolyze the hydrogen bond between poly chains to rupture the crystalline, but its hydrolytic activity cannot be detected alone (Ravindran and Jaiswal, 2015; Wilson 2012). The reducing sugar was not detected with the treated filter paper by CxEXL22 alone after $48 \mathrm{~h}$. It was found that the released reducing sugar was increased by 2.4-fold when the filter paper was treated with a mixture of CxEXL22 and cellulase for $12 \mathrm{~h}$ than that with both bovine serum albumin (BSA) and cellulase. It suggested that the CxEXL22 could synergistically hydrolyze cellulose with cellulose. Such synergistic action with the commercial cellulase was investigated using different substrates including microcrystalline cellulose (Avicel), cotton, filter paper, lignocellulose and $\alpha$-cellulose. As shown in Fig. 3B, CxEXL22 could 


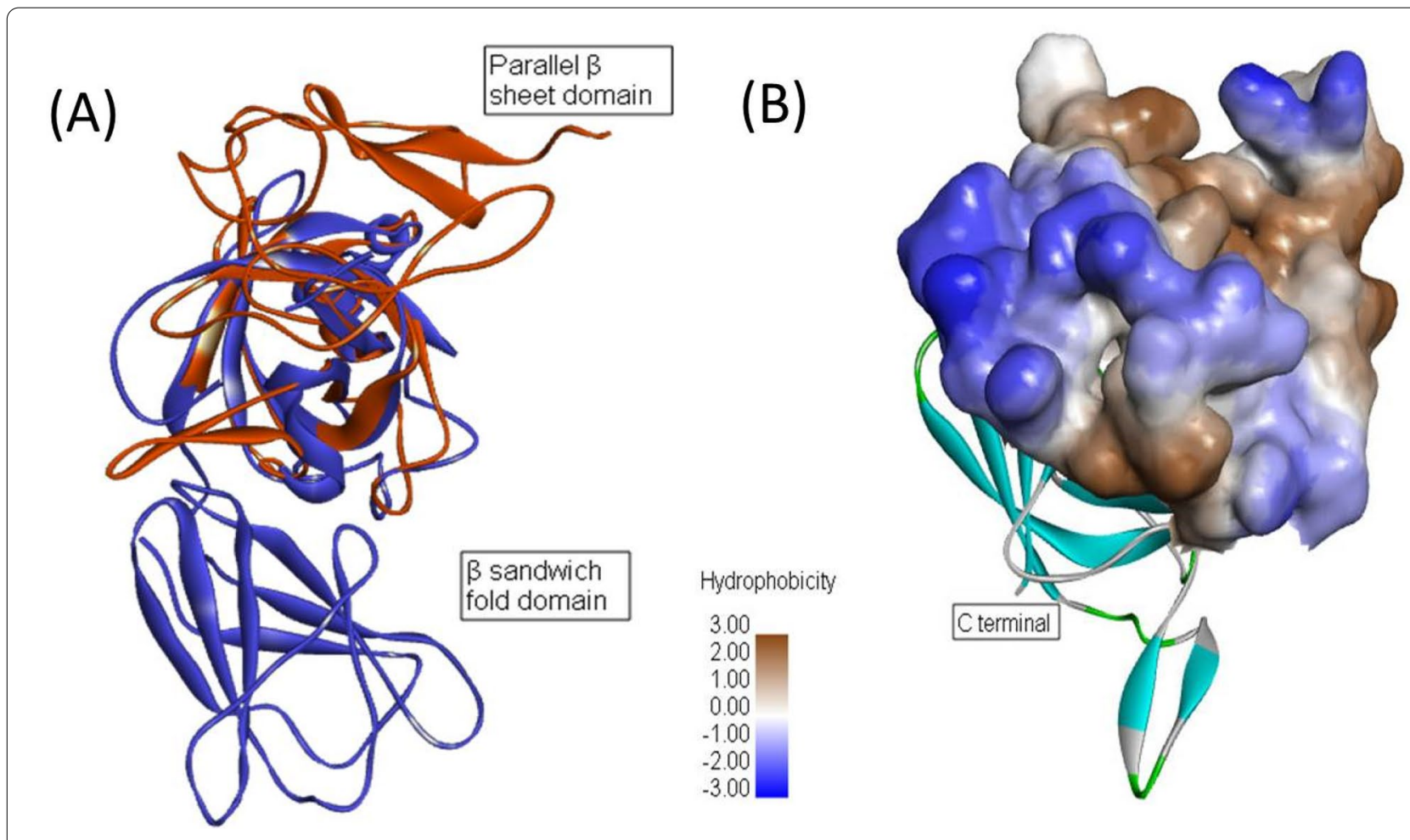

(C)

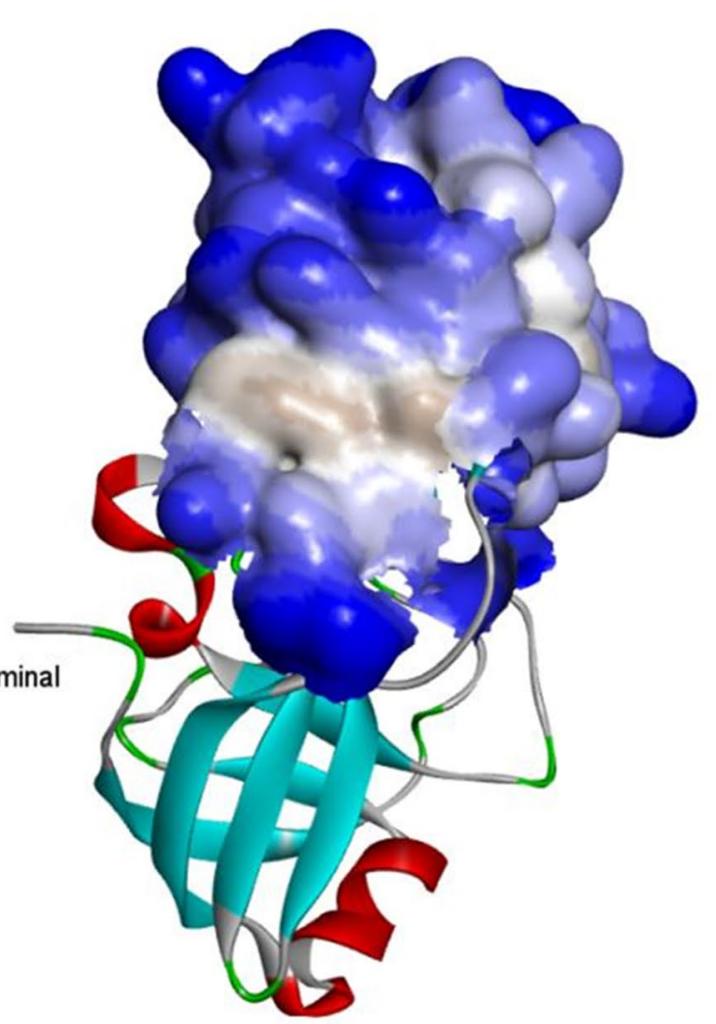

Fig. 2 The predicted structure model of CxEXL22: A overall structures superimposition of the predicted CxEXL22 modeling (orange) and BsEX (PDB:3D30, blue); $\mathbf{B}$ the hydrophobic groove surface of parallel $\beta$ sheet domain; $C$ the hydrophobic surface of BsExI 


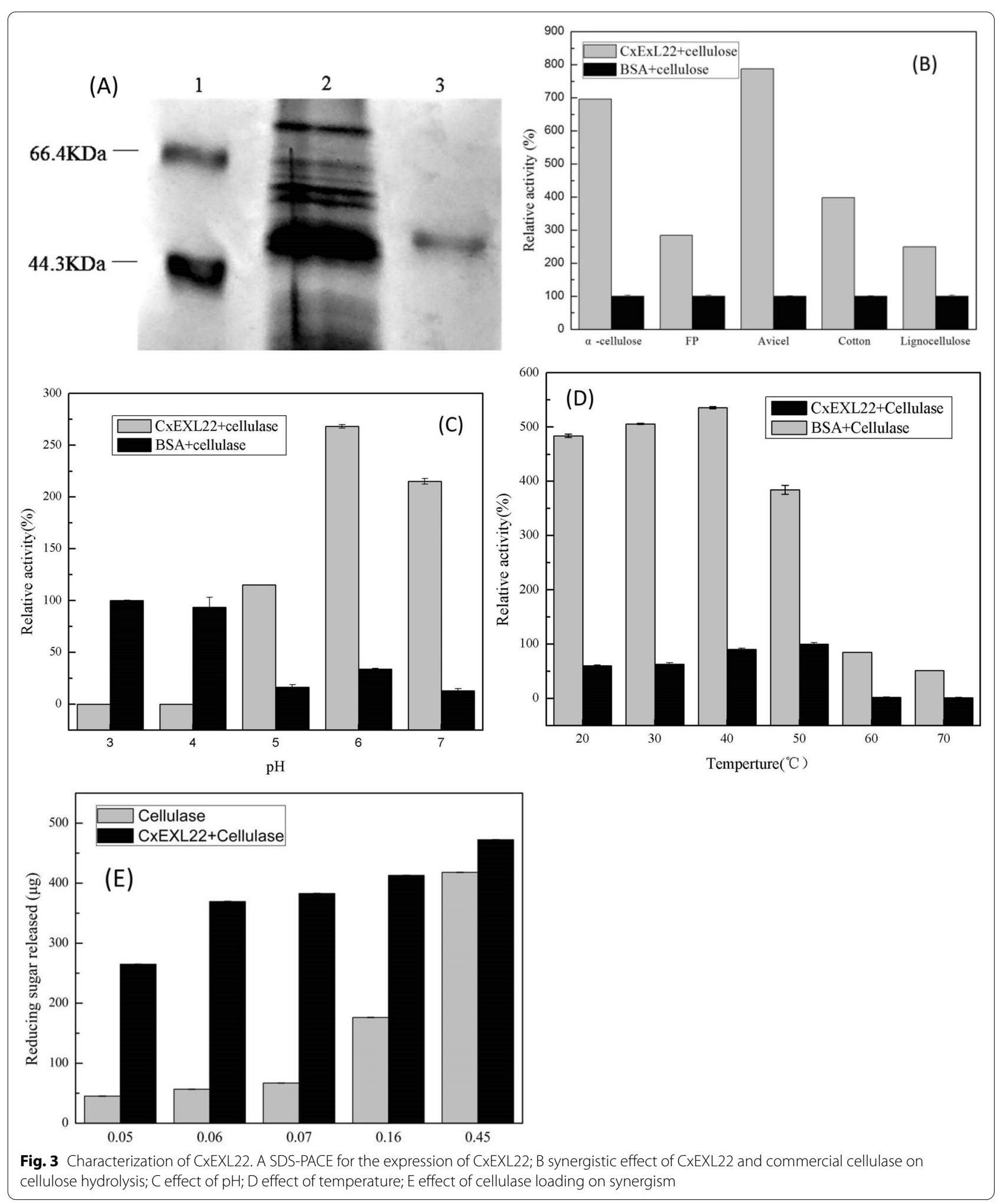


significantly increase the efficiency of cellulose hydrolysis. The hydrolytic efficiency of Avicel was enhanced by 7.9 times when treated with CxEXL22, whereas the relative activity of the treated filter paper and lignocellulose was only increased by 2.4 and 2.2 times, respectively. Avicel is a microcrystalline cellulose with a higher crystallinity than that of filter paper composed of both amorphous regions and crystalline fibers. It was reported that the expansin-like proteins show strongly synergism activity to filter paper than Avicel (Wang et al. 2014; Georgelis et al. 2014). Thus the CxEXL22 showed high specificity on crystalline cellulose, affected crystallinity and accessibility of cellulose to produce a synergistic effect with cellulase for cellulose hydrolysis.

\section{Optimization of CxEXL22 hydrolyzing cellulose}

The optimum hydrolysis conditions of CxEXL22 for disrupting the crystalline region were determined. As shown in Fig. 3C, cellulase gave a high activity at $\mathrm{pH} 3.0$ and 4.0 in the control with cellulase and bovine serum albumin, which is consistent with the optimum $\mathrm{pH}$ for the cellulase from Aspergillus niger (Hurst et al. 1977). However, the highest activity was obtained at $\mathrm{pH} 6.0$ when CxEXL22 synergistically cooperated with cellulase, which moved away from an original $\mathrm{pH}$. The $\mathrm{pH}$ dependence of CxEXL22 differed from those of previously reported and was inconsistent with the acid growth theory (Cosgrove 2000). In various plants, the plant cell wall grows faster when the wall $\mathrm{pH}$ is lowered below 5.5 and this phenomenon is known as acid growth (Dünser and Kleine-Vehn 2015). Expansins were isolated and identified to act as a catalyst to acid growth (Cosgrove 2000; Nakatani et al. 2013). This finding indicated that expansins had an acidic optimum to promote wall extension, which is similar to expansin-like proteins from microbes (Kim et al. 2009; Georgelis et al. 2014). It was of interest that no cellulase activity was detected to the CxEXL22-treated substrate at $\mathrm{pH}$ 3.0-4.0. Binding capability to the substrate depends on the $\mathrm{pH}$ of the incubation buffer (Liu et al. 2015). Expansins and cellulose compete for the limited binding sites on cellulose substrates (Kim et al. 2009; Liu et al. 2015). The result indicated the CxEXL22 may strongly bind to the polysaccharide substrate at acidic $\mathrm{pH}$ to seize the limited binding sites on cellulosic substrates, even completely inhibit the binding of cellulase. However, the CxEXL22 cannot depolymerize cellulose to expose much more microfibrils and enhances cellulose accessibility, resulting in cellulase cannot bind and hydrolase cellulose.

The hydrolysis activity of cellulase was set at $50{ }^{\circ} \mathrm{C}$ as the standard that is the reported optimal temperature (Hurst et al. 1977). However, the maximum synergistic activity of CxEXL22 with cellulase was achieved at $40{ }^{\circ} \mathrm{C}$, and it was eightfold greater than that of control under the same reaction temperature (Fig. 3D). When the temperature was raised to $70{ }^{\circ} \mathrm{C}$, it can still synergistically increase by twofold for cellulase activity although CxEXL22 activity was markedly lowered. This result indicated that CxEXL22 could be applied at a wide range of temperatures.

The cellulase loading is important for the synergism of expansin and cellulase to hydrolysis cellulose. As shown in Fig. 3E, the amount of reducing sugar released by cellulose hydrolysis increased significantly with the increase of the cellulase concentration from 0.05 to $0.45 \mathrm{U} / \mathrm{ml}$. But with the increase of cellulase loading, the synergistic effect became insignificant, which is consistent with the synergism of cellulase and BsEXLX1 (Kim et al. 2009). The synergistic effect is most significant at the low cellulase loading. Expansins and cellulase compete for the limited binding sites on substrates, resulting in a highly synergistic effect that occurs only at low concentrations of cellulase. Such low sugar yields in response to low cellulase loading are common when characterizing synergism between proteins and cellulases with CBM (Kim et al. 2009, 2014).

\section{Cellulose decrystallization by CxEXL22}

Crystallinity index was examined by XRD to evaluate the destruction of CxEXL22 on cellulose crystalline region. The crystallinity index was calculated with the height of four crystalline peaks based on the amorphous standard from XRD data (Park et al. 2010). Acid hydrolysis of cellulose can cause a conversion of the crystalline region to the amorphous region, thus the XRD spectra of Avicel and Avicel swelled by phosphoric acid indicated the change in the crystalline region (Fig. 4A). XRD spectrum showed changes in four peaks named as 101, 10i, 002 and 040, respectively, which were similar to those have been assumed in other reports (Ciolacu et al. 2011; Kim et al. 2013). As shown in Fig. 4B-D, the reduction in the height of these four crystallization peaks was also observed in different cellulose substrates treated with CxEXL22. Therefore, the rupture of the ordered crystal lattice assignments of cellulose was transformed into amorphous region after CxEXL22 treatment. Cellulose crystallinity index was calculated based on comparing the background intensity at $2 \theta=18^{\circ}$ with the peak height of 002 at around $22.8^{\circ}$. The crystallinity index of Avicel was decreased by $21 \%$ after treated with acid and $3.5 \%$ after treated with CxEXL22, which indicated that the ordered structure of the crystalline region was disrupted (Table 1). Moreover, a strong decrease (about 8.5\%) in the crystallinity degree was observed in the treated filter paper, presumably based on the accessibility of the filter paper, which contains much more terminal gaps between crystalline region and amorphous region for accessing by 

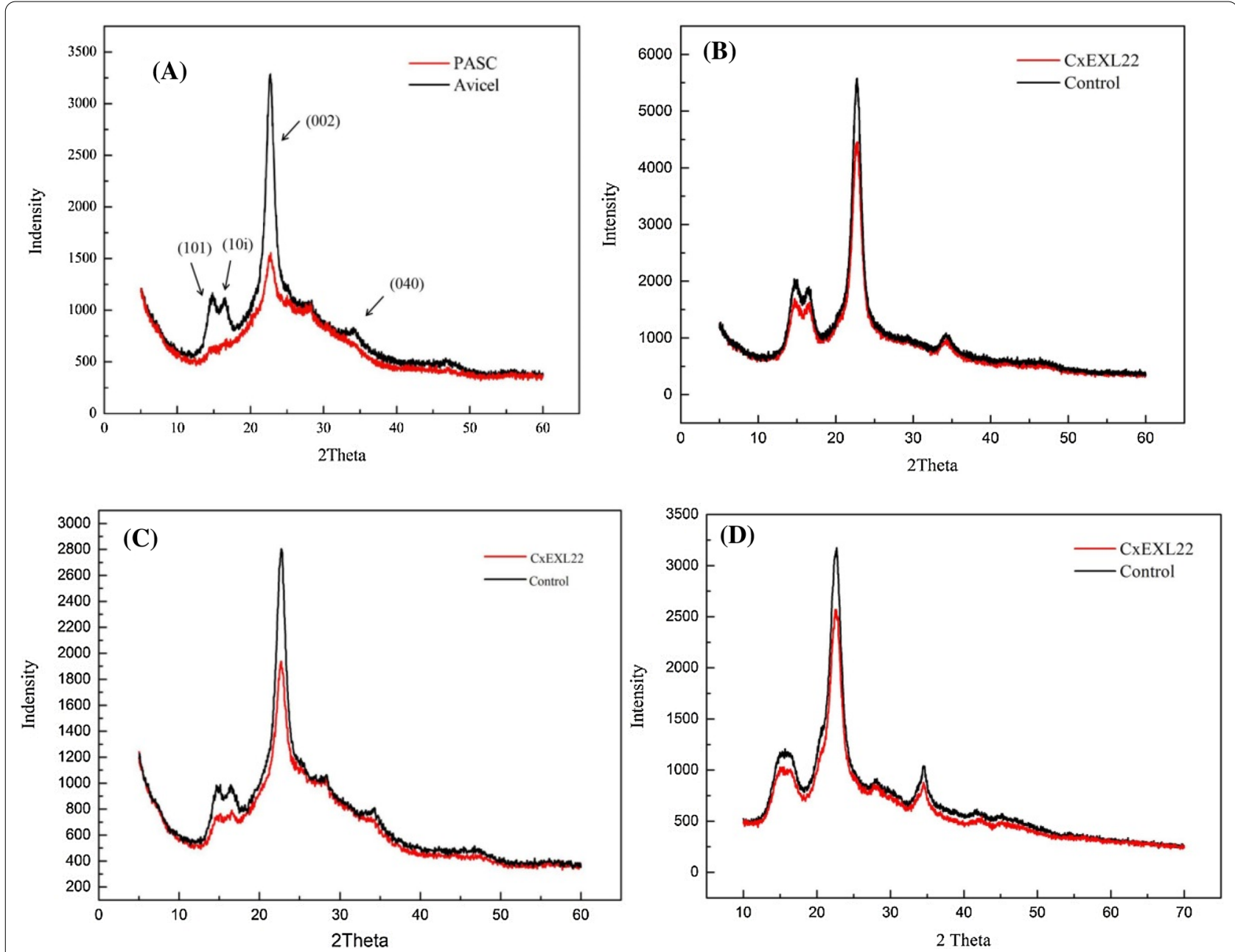

Fig. 4 X-ray diffraction spectrum of different cellulose substrates. A Phosphoric acid-swelling Avicel, B cotton, C filter paper and D Avicel

Table 1 Crystallinity index of CxEXL22-treated cellulose substrates, as determined by $\mathrm{X}$-ray diffraction

\begin{tabular}{llc}
\hline Cellulose substrates & \multicolumn{2}{l}{ Crystallinity index (\%) } \\
\cline { 2 - 3 } & Untreated & Treated \\
\hline Phosphoric acid-swelling Avicel & 75.35 & 54.32 \\
Avicel & 74.79 & 71.30 \\
Cotton & 82.04 & 78.12 \\
Filter paper & 70.64 & 62.12 \\
\hline
\end{tabular}

CxEXL22. In conclusion, CxEXL22 could significantly reduce cellulose crystallinity that will cause subsequent depolymerization of the crystalline region.

Structure of Avicel treated with CxEXL22 was analyzed by FTIR. The band near $3300 \mathrm{~cm}^{-1}$ can be attributed to $\mathrm{O}-\mathrm{H}$ stretching vibrations (Ciolacu et al. 2011), which showed higher intensity than that of control (Fig. 5).

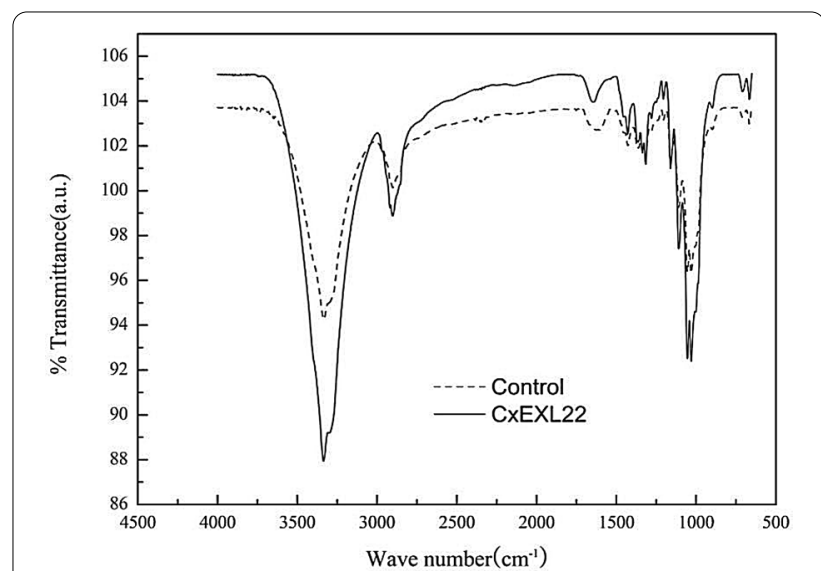

Fig. 5 Comparison of Fourier-transform infrared spectra of Avicel treated with CXEXL22 with that of untreated Avicel 

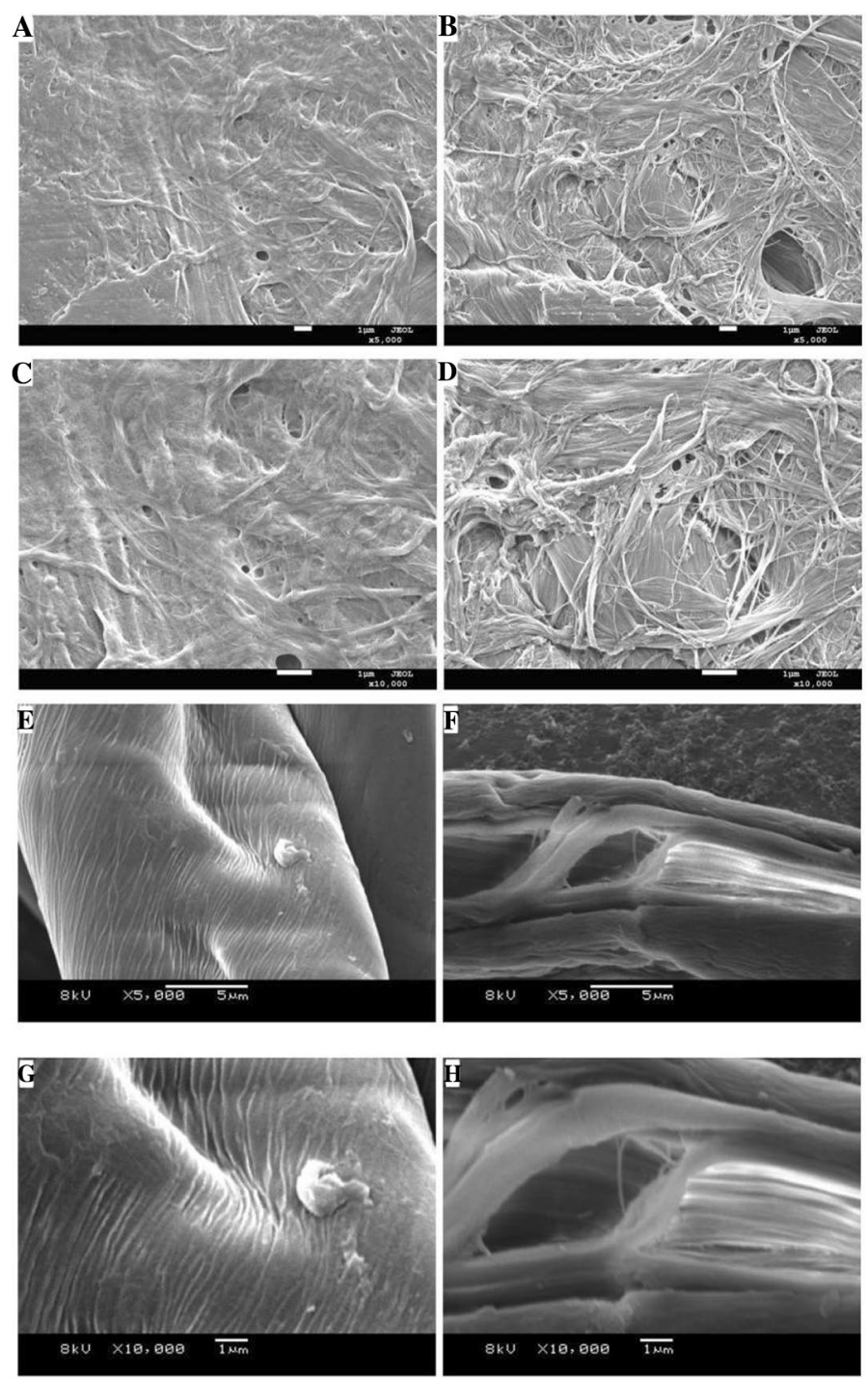

Fig. 6 Scanning electron microscopy observation of different cellulose substrates: A, C ddH $\mathrm{H}_{2} \mathrm{O}$-treated filter paper at A $5000 \times$ and $\mathbf{C} 10,000 \times$; B, D CXEXL22-treated filter paper at B $5000 \times$ and $\mathbf{D} 10,000 \times$; E, $\mathbf{G}$ dd H ${ }_{2} \mathrm{O}$-treated cotton at $\mathbf{E} 5000 \times$ and $\mathbf{G} 10,000 \times$; and $\mathbf{F}$, $\mathbf{H}$ CxEXL22-treated cotton at $\mathbf{F} 5000 \times$ and $\mathbf{H} 10,000 \times$

It suggested the rupture of intra- and inter-molecular hydrogen bonds in the treated Avicel that resulted in the exposure of much more $-\mathrm{OH}$ groups (Ciolacu et al. 2011; Kim et al. 2013). The skeletal stretching of intramolecular $\mathrm{C}-\mathrm{OH}$ is near $1030 \mathrm{~cm}^{-1}$. As shown in Fig. 5, the absorption of $\mathrm{O}-\mathrm{H}$ stretching of the treated Avicel was intensified, which reflected the entrance of water molecules causing the exposure of more hydrogen bonds of networks in the crystalline region compared with the untreated Avicel. This result was well supported by the XRD observation above, which confirmed that the hydrogen bond between cellulose chains was hydrolyzed by CxEXL22 to expose more $\mathrm{O}-\mathrm{H}$ groups between the chains and the $\mathrm{O}-\mathrm{H}$ of the entered water molecules in polymers. This caused the decrystallization of the crystalline region leading to the depolymerization of cellulose.

\section{Cellulose depolymerization by CxEXL22}

To visualize the effect of CxEXL22 on cellulose polymer, microphotography of different polysaccharide substrates was performed using SEM. As suggested that macrofibril was the agglomeration of microfibrils (Chinga-Carrasco 2011), the surface of untreated filter paper showed macrofibrils with high density and overlapping, whereas the CxEXL22-treated filter paper showed dispersed microfibers (Fig. 6). Cotton fiber 
in the untreated control was smooth and flat, but the CxEXL22-treated cotton fiber showed microfibril bundles and loosened intermolecular surface between macrofibrils, which caused cellulose decrystallization and depolymerization. Such results suggested that the dense structure of crystalline region was broken and the looseness of cellulose was significantly increased after treated with CxEXL22. The binding of cellulose and expansin-like protein is entropy-driven triggered by the hydrophobic interactions between the cellulose and the hydrophobic flat in D2 (Georgelis et al. 2012). We deduced that the CxEXL22 binds to the macrofibrils by the hydrophobic surface of the parallel $\beta$-sheet domain, and slides on the cellulose chains to hydrolyze intermolecular and intramolecular hydrogen bonds tightly packed cellulose chains. Therefore, the agglomeration of microfibrils was distinctly decreased by CxEXL22 and the macrofibril was depolymerized to expose much more microfibrils and increase cellulose accessibility.

\section{Conclusion}

The crystallinity and polymerization of cellulose is the key bottleneck to the efficiency of cellulose degradation. In this context, a novel expansin-like protein CxEXL22 caused cellulose decrystallization and exhibited effective synergistical catalytic activity against cellulose with cellulase. The direct phylogenetic relationship of CxEXL22 implied that genetic transfers occurred between nematode and nematicidal fungi. The result implied that CxEXL22 strongly binds to cellulose chain via the hydrophobic groove surface of parallel $\beta$-sheet domain and breaks up hydrogen bonds between cellulose chains. Consequently, CxEXL22 splits cellulose chains depolymerizing cellulose to expose much more microfibrils, thus enhancing cellulose accessibility.

\section{Supplementary Information}

The online version contains supplementary material available at https://doi. org/10.1186/s40643-021-00446-7.

Additional file 1: Table S1. Expansin-like proteins from plants, nematodes and microbes used for the CXEXL22 phylogenetic analysis. Fig. S1. Conserved domains of CXEXL2. Fig. S2. The sequence alignment of CXEXL22 with modeling temple. Fig. S3. SEM images of (A) the original filter paper and (B) the phosphoric acid treated filter paper. Fig. S4. Structure alignment RMSD values of (1) the predicted structure of CXEXL22 protein with the Phyre2 web server (2) the predicted structure of CXEXL22 protein with the I-TASSER server and (3) the predicted structure of CXEXL22 protein with the Swiss-Model server

\section{Authors' contributions}

Data curation, YS, YZ, JG, LY and WT; formal analysis, RL; funding acquisition, RL; investigation, RL; methodology, LY and FY; software, YZ, LY and WT; supervision, $\mathrm{RL}$ and $\mathrm{XL}$; validation, RL; writing-original draft, RL and XL. All authors read and approved the final manuscript.

\section{Funding}

This work greatly acknowledges the financial support by the National Natural Science Foundation of China (No. 31600640), Science and technology Department of Dalian (2020RQ118) and Liaoning province Education Sciences Foundation (No. J2019015).

\section{Availability of data and materials}

The dataset (graphs and tables) supporting the conclusions of this article are available.

\section{Declarations}

Ethics approval and consent to participate

Not applicable.

Consent for publication

Not applicable.

\section{Competing interests}

All authors declare that they have no conflict of interest.

Received: 13 July 2021 Accepted: 16 September 2021

Published online: 24 September 2021

\section{References}

Arantes V, Saddler JN (2010) Access to cellulose limits the efficiency of enzymatic hydrolysis: the role of amorphogenesis. Biotechnol Biofuels 3(1):4. https://doi.org/10.1186/1754-6834-3-4

Chen L, Wang Q, Hirth K, Baez C, Agarwal UP, Zhu J (2015) Tailoring the yield and characteristics of wood cellulose nanocrystals (CNC) using concentrated acid hydrolysis. Cellulose 22(3):1753-1762. https://doi. org/10.1007/s10570-015-0615-1

Chinga-Carrasco G (2011) Cellulose fibres, nanofibrils and microfibrils: the morphological sequence of MFC components from a plant physiology and fibre technology point of view. Nanoscale Res Lett 6:417. https:// doi.org/10.1186/1556-276X-6-417

Ciolacu D, Ciolacu F, Popa VI (2011) Amorphous cellulose-structure and characterization. Cell Chem Technol 45:13-21. http://www.cellulosec hemtechnol.ro/pdf/CCT1-2(2011)/p.13-21.pdf.

Cosgrove DJ (2000) Loosening of plant cell walls by expansins. Nature 407:321-326. https://doi.org/10.1038/35030000

Cosgrove DJ (2015) Plant expansins: diversity and interactions with plant cell walls. Curr Opin Plant Biol 25:162-172. https://doi.org/10.1016/j. pbi.2015.05.014

Cosgrove DJ (2017) Microbial expansins. Annu Rev Microbiol 71:479-497. https://doi.org/10.1146/annurev-micro-090816-093315

Dünser K, Kleine-Vehn J (2015) Differential growth regulation in plants-the acid growth balloon theory. Curr Opin Plant Biol 28:55-59. https://doi. org/10.1016/j.pbi.2015.08.009

Georgelis N, Yennawar NH, Cosgrove DJ (2012) Structural basis for entropydriven cellulose binding by a type-A cellulose-binding module (CBM) and bacterial expansin. P Natl Acad Sci USA 109(37):14830-14835. https://doi.org/10.1073/pnas.1213200109

Georgelis N, Nikolaidis N, Cosgrove DJ (2014) Biochemical analysis of expansin-like proteins from microbes. Carbohyd Polym 100:17-23. https://doi.org/10.1016/j.carbpol.2013.04.094

Georgelis N, Nikolaidis N, Cosgrove DJ (2015) Bacterial expansins and related proteins from the world of microbes. Appl Microbiol Biotechnol 99:3807-3823. https://doi.org/10.1007/s00253-015-6534-0

Guo H, Chang Y, Lee DJ (2018) Enzymatic saccharification of lignocellulosic biorefinery: research focuses. Bioresource Technol 252:198-215. https://doi.org/10.1016/j.biortech.2017.12.062

Hurst PL, Nielsen J, Sullivan PA, Shepherd M (1977) Purification and properties of a cellulase from Aspergillus niger. Biochem J 165:33-41. https:// doi.org/10.1042/bj1650033 
Kelley LA, Mezulis S, Yates CM, Wass MN, Sternberg MJ (2015) The Phyre2 web portal for protein modeling, prediction and analysis. Nat Protoc 10(6):845. https://doi.org/10.1038/nprot.2015.053

Kerff F, Amoroso A, Herman R, Sauvage E, Petrella S, Filée P, Charlier P, Joris B, Tabuchi A, Nikolaidis N (2008) Crystal structure and activity of Bacillus subtilis YoaJ (EXLX1), a bacterial expansin that promotes root colonization. PNAS 105(44):16876-16881. https://doi.org/10.1073/pnas.08093 82105

Kim ES, Lee HJ, Bang WG, Choi IG, Kim KH (2009) Functional characterization of a bacterial expansin from Bacillus subtilis for enhanced enzymatic hydrolysis of cellulose. Biotechnol Bioeng 102:1342-1353. https://doi.org/ 10.1002/bit.22193

Kim SH, Lee CM, Kafle K (2013) Characterization of crystalline cellulose in biomass: basic principles, applications, and limitations of XRD, NMR, IR, Raman, and SFG. Korean J Chem Eng 30:2127-2141. https://doi.org/10. 1007/s11814-013-0162-0

Kim IJ, Lee HJ, Choi IG, Kim KH (2014) Synergistic proteins for the enhanced enzymatic hydrolysis of cellulose by cellulase. Appl Microbiol Biot 98(20):8469-8480. https://doi.org/10.1007/s00253-014-6001-3

Kuo CH, Lee CK (2009) Enhancement of enzymatic saccharification of cellulose by cellulose dissolution pretreatments. Carbohyd Polym 77:41-46. https://doi.org/10.1016/j.carbpol.2008.12.003

Lan W, Zhang H, Chen X, Yang F, Li X (2016) Gelatinization and decrystallization of cellulose by newly isolated Arthrobotrys sp. CX1 to facilitate cellulose degradability. Cellulose 23:3543-3554. https://doi.org/10.1007/ s10570-016-1092-x

Lee H, Lee S, Ko H, Kim K, Choi I-G (2010) An expansin-like protein from Hahella chejuensis binds cellulose and enhances cellulase activity. Mol Cells 29(4):379-438. https://doi.org/10.1007/s10059-010-0033-z

Letunic I, Khedkar S, Bork P (2021) SMART: recent updates, new developments and status in 2020. Nucleic Acids Res 49(1):458-460. https://doi.org/10. 1093/nar/gkaa937

Lindman B, Karlström G, Stigsson L (2010) On the mechanism of dissolution of cellulose. J Mol Liq 156(1):76-81. https://doi.org/10.1016/j.molliq.2010. 04.016

Liu X, Liu C, Ma Y, Hong J, Zhang M (2014) Heterologous expression and functional characterization of a novel cellulose-disruptive protein LeEXP2 from Lycopersicum esculentum. J Biotechnol 186:148-155. https://doi. org/10.1016/j.jbiotec.2014.07.013

Liu X, Ma Y, Zhang M (2015) Research advances in expansins and expansionlike proteins involved in lignocellulose degradation. Biotechnol Lett 37(8):1541-1551. https://doi.org/10.1007/s10529-015-1842-0

Nakatani Y, Yamada R, Ogino C, Kondo A (2013) Synergetic effect of yeast cell-surface expression of cellulase and expansin-like protein on direct ethanol production from cellulose. Microb Cell Fact 12(1):66. https://doi. org/10.1186/1475-2859-12-66

Nikolaidis N, Doran N, Cosgrove DJ (2013) Plant expansins in bacteria and fungi: evolution by horizontal gene transfer and independent domain fusion. Mol Bio Evol 31(2):376-386. https://doi.org/10.1093/molbev/ mst206

Park S, Baker JO, Himmel ME, Parilla PA, Johnson DK (2010) Cellulose crystallinity index: measurement techniques and their impact on interpreting cellulase performance. Biotechnol Biofuels 3:10. https://doi.org/10.1186/ 1754-6834-3-10
Ragauskas AJ, Williams CK, Davison BH, Britovsek G, Cairney J, Eckert CA, Frederick WJ, Hallett JP, Leak DJ, Liotta CL (2006) The path forward for biofuels and biomaterials. Science 31:484-489. https://doi.org/10.1126/ science. 1114736

Ravindran R, Jaiswal AK (2015) A comprehensive review on pre-treatment strategy for lignocellulosic food industry waste: challenges and opportunities. Bioresour Technol 199:92-102. https://doi.org/10.1016/j.biortech. 2015.07.106

Rinaldi R, Palkovits R, Schüth F (2008) Depolymerization of cellulose using solid catalysts in ionic liquids. Angew Chem Int Edit 47:8047-8050. https://doi. org/10.1002/anie.200802879

Sabbadin F, Hemsworth GR, Ciano L, Henrissat B, Dupree P, Tryfona T, Marques RDS, Sweeney ST, Besser K, Elias L, Pesante G, Li Y, Dowle AA, Bates R, Gomez LD, Simister R, Davies GJ, Walton PH, Bruce NC, McQueen-Mason SJ (2018) An ancient family of lytic polysaccharide monooxygenases with roles in arthropod development and biomass digestion. Nat Commun 9(1):756. https://doi.org/10.1038/s41467-018-03142-x

Sharma HK, Xu C, Qin W (2019) Biological pretreatment of lignocellulosic biomass for biofuels and bioproducts: an overview. Waste Biomass Valori 10(2):235-251. https://doi.org/10.1007/s12649-017-0059-y

Tadesse H, Luque R (2011) Advances on biomass pretreatment using ionic liquids: an overview. Energ Environ Sci 4(10):3913-3929. https://doi.org/ 10.1039/c0ee00667j

Tamura K, Stecher G, Peterson D, Filipski A, Kumar S (2013) MEGA6: molecular evolutionary genetics analysis version 6. Mol Bio Evol 30(12):2725-2729. https://doi.org/10.1093/molbev/mst197

Van Den Ent F, Löwe J (2006) RF cloning: a restriction-free method for inserting target genes into plasmids. J Biochem Bioph Meth 67:67-74. https://doi. org/10.1016/j.jbbm.2005.12.008

Villares A, Moreau C, Bennati-Granier C, Garajova S, Foucat L, Falourd X, Saake B, Berrin JG, Cathala B (2017) Lytic polysaccharide monooxygenases disrupt the cellulose fibers structure. Sci Rep 7:40262. https://doi.org/10. 1016/j.tplants.2016.07.012

Wang W, Liu C, Ma Y, Liu X, Zhang K, Zhang M (2014) Improved production of two expansin-like proteins in Pichia pastoris and investigation of their functional properties. Biochem Eng J 84:16-27. https://doi.org/10.1016/j. bej.2013.12.018

Wilson DB (2009) Cellulases and biofuels. Curr Opin Biotech 20:295-299. https://doi.org/10.1016/j.copbio.2009.05.007

Wilson DB (2012) Processive and nonprocessive cellulases for biofuel production—lessons from bacterial genomes and structural analysis. Appl Microbiol Biot 93(2):497-502. https://doi.org/10.1007/s00253-011-3791-9

Zhang YP, Hong J, Ye X (2009) Cellulase assays. In: Mielenz J (ed) Biofuels. Humana Press, Totowa, NJ, pp 213-231. https://doi.org/10.1007/978-160761-214-8_14

\section{Publisher's Note}

Springer Nature remains neutral with regard to jurisdictional claims in published maps and institutional affiliations.

\section{Submit your manuscript to a SpringerOpen ${ }^{\circ}$ journal and benefit from:}

- Convenient online submission

- Rigorous peer review

- Open access: articles freely available online

- High visibility within the field

- Retaining the copyright to your article

Submit your next manuscript at $\boldsymbol{\nabla}$ springeropen.com 